\title{
9 Gewaltaktionen und gesellschaftlicher Prozess
}

Nach Meinung des amerikanischen Soziologen und Zukunftsforschers Sennett (2000) müssen wir uns darauf einstellen, dass sich das Verhältnis von privatem und öffentlichem Leben in den nächsten Jahren wesentlich verändern wird. Er thematisiert das "Verhältnis von Terror und Moderne“ und stellt die Frage, zu welchen Auswirkungen Massaker und Terrorangriffe auf unser Verständnis über Kultur und Zivilisation führen. Er geht davon aus, dass Massaker und Terror und die damit verbundenen Schrecken und Ängste ein immanenter Bestandteil der Moderne werden, insbesondere auch die Erfahrung von Entwurzelung und plötzlich eintretender Verstörung, verbunden mit einem Verlust des Sicherheitsgefühls.

Die Deutsche Gesellschaft für Psychoanalyse, Psychotherapie, Psychosomatik und Tiefenpsychologie (DCPT) wählte für ihre 52. Jahrestagung im September 2001 bewusst das Thema "Gewalt und Zivilisation“. Die meisten Referenten waren der Meinung, dass die von den Tätern bewirkte Traumatisierung bei dem Ereignis vom 11.9.2001 in New York beabsichtigt gewesen war, um die Symbole westlicher Übermacht zu attackieren und die Ohnmacht in der „Dritten Welt“ zu verdeutlichen.

Die Psychoanalytiker äußerten ihre Befremdung über die Tatsache, dass die Politik sehr schnell bereit war, rechtsstaatliche Mittel zugunsten eines Kampfes der "Guten“ gegen die „Bösen“ aufzugeben. Sie appellierten an die Politik, den „Kreislauf gegenseitiger Kränkungen“ nicht fortzusetzen, sondern sich um die Wiederherstellung des Dialogs auf allen gesellschaftlichen Ebenen zu bemü- 
hen. Dies beinhalte auch einen eventuellen Verzicht auf Dominanzansprüche und nicht deren Durchsetzung. Nur so könne die Entstehung weiterer Traumatisierungen des kollektiven Selbstgefühls infolge narzisstischer Kränkungen mit tödliche Reaktionen verhindert werden, die letztlich nur weitere zahlreiche Opfer zur Folge hätten. Die „Stimme der Vernunft“ müsse laut und vernehmlich gegen Kriegsgeschrei und Vergeltungssucht Position beziehen (Dt. Ärzteblatt, 2001).

Strasser (2001) beschreibt eine nachlassende Bindekraft zwischen Familie, Schule und den ihnen anvertrauten Kindern und Jugendlichen. Damit gehe eine zunehmende Entsolidarisierung und Auflösung zwischenmenschlicher Bindungen einher. Strasser weist daraufhin, dass die aktive Lebensgestaltung durch das autonome Individuum nicht mehr als „Lebensideal“ gilt, sondern die umfassende „Selbstinstrumentalisierung für heteronome Zwecke“ und ein „Leben im Passiv“. Seiner Meinung nach kann das eigene Leben nur dann sinnvoll sein, wenn man es in einen "das eigene Ich umgreifenden Kontext“ stellen kann. Um sinnvoll und frei leben zu können, müssten Jugendliche heute wissen, was die eigene Berufung oder Bestimmung ist oder, was sie als eigene Berufung oder Bestimmung erkennen und anerkennen, statt sich immer mehr in ,hektische Anpassungsleistungen " zu verstricken. Sonst verstärke sich das Gefühl, das „falsche Leben“ zu leben. Ansonsten würde die eigene Verortung, die interne und äußere Kohärenz drohen verlorenzugehen. Die „Spirale der Gewalt“ beginnt mit einer „Spirale der gestörten Kommunikation“, die dann dauerhaft zum Abbruch der Kommunikation führt.

In der kommunikativen Alltagspraxis in der Familie und Schule muss sich ein „Vertrauenskapital“ bilden können. Freiheit bemisst sich jedoch nach dem Grad, in dem wir fähig sind, die Kontrolle über Kräfte, die sonst uns selbst kontrollieren würden zu erlangen (Habermas und Derrida 2006).

Keupp et al. (2008) beschreiben zehn gesellschaftliche Umbrucherfahrungen in spätmodernen Gesellschaften.

\section{Gesellschaftliche Umbruchserfahrungen nach Keupp et al. (2008)}

- Jugendliche fühlen sich dann „entbettet“, wenn sie in ihrer individuellen Lebensführung kaum „kulturelle Korsettstangen“ in ihrem Life-Style-Konzept nutzen können.

- Die noch von früheren Generationen vermittelten Vorstellungen von Erziehung, Sexualität, Gesundheit, Geschlechter- oder Generationenbeziehung verlieren den Charakter des Selbstverständlichen und ihre „Prägekraft“.

- Die Erwerbsarbeit als Basis der Identitätsbildung wird zunehmend brüchig. "Soziale Einbindungen“, in denen soziale Anerkennung erfahren werden kann, sind schwieriger geworden.

- Es stürmt auf die heutigen Jugendlichen eine ungeheuer schnell wachsende Vielfalt von Wünschen, Optionen, Gelegenheiten, Verpflichtungen und 
Werten ein. Sie müssen damit leben, dass vieles höchst widersprüchlich ist, sodass hohe psychische "Spaltungskompetenzen“ gefordert sind.

- Es besteht die Gefahr von „Kommunikationsrissen“ und Parallelwelten zwischen Eltern und Kindern, die sich heute souverän in virtuellen Welten bewegen.

- Das Zeitgefühl erfährt eine „Gegenwartsschrumpfung durch Innovationsverdichtung“ mit einer Verringerung der „Halbwertszeiten“ des aktuell geltenden Wissens.

- Da die Konventionen immer weniger „Selbstverständlichkeiten“ sind, kann der Einzelne nicht mehr auf fest etablierte Verhaltens- und Denkmuster zurückgreifen. Sein Leben wird zu einem „Projekt“.

- Es gibt neue Varianten von Familienformen wie Patchwork-Familien.

- Es gibt Veränderungen der Geschlechterrollen.

- Die zunehmende Individualisierung hat das Verhältnis vom Einzelnen zur Gemeinschaft verändert, und es droht eine Auflösung von traditionellen Strukturen.

\section{Der Verlust des Glaubens an „Meta-Erzählungen“ erzeugt einen indivi- dualisierten „Sinn-Bastler“. Der Einzelne wird zum Konstrukteur seines eigenen Sinnsystems.}

„Aus Fehlern lernen“ ist ein wichtiges pädagogisches Prinzip. Im Gegensatz dazu existiert heute keine „Kultur des Scheiterns“ mehr. Die Umsetzung eines Lehrplans ist meist wichtiger als die „Kultur des Lernens“ zu lernen und zeitgemäßere Lernformen und Settings zu nutzen.

Alle heutigen Aggressionstheorien, seien sie lerntheoretisch, neurobiologisch, soziologisch oder tiefenpsychologisch begründet, sind genötigt, zu den unterschiedlichsten Deutungssystemen von Gewalt „Brücken“ zu schlagen. So sollen sie den biologischen Grundlagen, den soziologischen Bedingungen, den gesellschaftlichen Normen, den verinnerlichten Wertschätzungen, den kognitiven Deutungssystemen, den gespeicherten Handlungsmustern, den Erwartungen und den Glaubenssystemen angemessen Rechnung tragen. Diese Komplexität macht es heutigen jungen Leuten schwer, darauf angemessene Antworten und Verhaltensweisen zu entwickeln. Gescheiterte Integrationsbemühungen beinhalten aber die Gefahr der Gewalttätigkeit, die nach Arendt (2000) in allen Bereichen des Lebens in unseren westlichen Gesellschaften vorhanden ist. Deshalb sind nach Meinung von Levin und Fox (2001) rein „technische“ Maßnahmen zur Reduzierung von School Shootings wenig sinnvoll. Die Installation von Metalldetektoren und Überwachungskameras in Schulen und eine „Null-Toleranz-Strategie“ konnten bisher nicht zu einer Reduzierung des Problems beitragen. Sie empfehlen daher eine Verringerung der Klassengrößen, die Erweiterung von Angeboten außerhalb des Lehrplans und ein AntiAggressions-Training. 
Vorfälle von schwerer zielgerichteter Gewalt von jungen Menschen gegen Mitschülerinnen und Mitschüler, Lehrkräfte und Schulpersonal werden sich trotz aller Bemühungen in Zukunft nicht gänzlich verhindern lassen. Deshalb ist es Aufgabe aller am Schulsystem Beteiligter, weiterhin mit großer Sensibilität derartigen Entwicklungen entgegenzutreten und Gewalttaten möglichst vorzubeugen.

Medienberichte über Amokläufe können zu weiteren School Shootings anregen. Das wurde bei der Analyse einiger Schul-Amokläufe deutlich, als man die Internetnutzung der Amokläufer vor ihrer Taten ermittelte. So ist auch verständlich, dass zwischen den Amokläufen an verschiedenen Orten eine Verbindung besteht, indem sie sehr nahe aufeinanderfolgten. So ging dem Amoklauf an der Schule in Winnenden acht Stunden zuvor ein Amoklauf in den USA voraus.

Andererseits führt die breite Medienberichterstattung auch zu einer „Sensibilisierung" der Öffentlichkeit und vermehrt zu kinder- und jugendpsychiatrischen Untersuchungen an Schülern, sodass die Möglichkeit zeitnaher präventiver Maßnahmen gegeben ist und damit auch weitere geplante School Shootings verhindert werden können.

Es ist wichtig, dass sich Therapeuten mit den gesellschaftlichen und sozialen Veränderungen auseinandersetzen. Dies beinhaltet, von der Annahme einseitiger Entstehungsbedingungen wegzukommen.

\subsection{Was ist zu tun?}

Da es aufgrund der Leistungsverdichtung und des daraus folgenden Drucks und Stresses immer mehr schwierige Schüler in Zukunft geben wird, werden auf die Schulen mehr sozialpädagogische Aufgaben der Schule zukommen (Bohnsack und Leber 1996). Wichtig sind vor allem ein ständiger Austausch und eine Vernetzung von Schule mit Polizei, Schulpsychologischem Dienst, Kinder- und Jugendpsychiatern, Kinder- und Jugendlichentherapeuten, Erziehungsberatungsstellen und Jugendhilfe. Dabei sind insbesondere Schulpsychologen und Schulsozialarbeiter „Schlüsselfiguren“, die verzweifelten oder resignierenden Schülern Perspektiven innerhalb von Schule, Familie und Gesellschaft aufzeigen können.

Ein gutes Klassenklima und Lehrer mit Vorbildfunktion, ein enger Austausch zwischen Lehrern und Eltern sind von unschätzbarem Wert, wenn genügend Zeitressourcen dafür zur Verfügung stehen. Meist wird jedoch vonseiten der Lehrer der übervolle Lehrplan bei verkürzter Schulzeit ins Feld geführt. Schüler müssen auch im Rahmen des Unterrichts zu einem kritischen Umgang mit Gewalt verherrlichenden Inhalten erzogen und die Eltern miteinbezogen werden. 
Das von W. Huck in LWL - Universitätsklinik Kinder- und Jugendpsychiatrie Hamm - durchgeführte „Psychoedukationsprogramm für Eltern und Jugendliche“ (2007) und das „Tagesklinische Modellprojekt für mediensüchtige und gefährdete Jugendliche“ (2008) machte deutlich, dass Eltern und Jugendliche oft in Parallelwelten leben und kaum Zeit für einen gemeinsamen Austausch oder gemeinsame Aktionen haben. Nur durch eine intensive Kommunikation zwischen Kindern, Eltern und Schule kann verhindert werden, dass sich Jugendliche in eine destruktive Fantasiewelt zurückziehen, um aus einer für sie unerträglichen und perspektivlos gewordenen Realität zu fliehen.

\subsubsection{Risiko für Trittbrettfahrer und „Sensation seeker“}

Schüler, die mit einer Spielzeugpistole auf Schulhöfen spektakuläre Bedrohungssituationen inszenieren, müssen zukünftig damit rechnen, für den Polizeieinsatz und für die Prozesskosten zu zahlen, auch wenn es nur eine ,jugendliche Dummheit“ war. Bei einer Alarmierung der Polizei und anderer Rettungskräfte wird ein Routineprogramm - oftmals unter Beteiligung des SEKgestartet, weil die Polizei hundertprozentig sichergehen muss, um alle Risiken zu minimieren.

\subsubsection{Spezifische Präventionsprogramme}

Generell müssen Lehrerinnen und Lehrer in der Aus- und Fortbildung auf eine veränderte Schüler-Klientel vorbereitet werden. Dabei kann es hilfreich sein, die systemischen Zusammenhänge der Beziehungen in der Schulklasse und Schule und seine Hintergründe wie ungelöste familiäre Konflikte zu verstehen, die immer wieder zu Reinszenierungen und zur Verlagerung des „Kampffeldes" in die Schule hineinwirken.

Regelmäßige Schulungen zur Deeskalation für alle Lehrkräfte und Einstimmungen auf Krisenlagen sind notwendig, um sich intensiv mit dem von den Schulministerien entwickelten „Notfallordnern“ auseinanderzusetzen. Hinzu kommen spezifische Schulungen von Krisenteams.

Mittlerweile existiert eine Reihe von spezifischen Präventionsprogrammen, wie das „Faustlos-Programm“ von Cierpka (2005) und das Freiburger „Anti-GewaltTraining(FACT)“von Fröhlich-Gildhoff (2006). Sie bringen Kindergärten, Grundund weiterführende Schulen in die Lage, zukünftige kriminelle Aktionen rechtzeitig zu verhindern. Durch diese Programme zur Gewaltprävention in Schulen können sehr viele Kinder - vor allem Kinder aus belasteten Familien erreicht werden. Dabei ist das Ziel, Stigmatisierungsprozesse zu vermeiden. Eine Vielzahl dieser Maßnahmen wurde bereits evaluiert, und es konnte gezeigt werden, dass die verschiedenen Präventionsansätze zu signifikanten positiven Effekten führen. Präventionskonzepte sind langfristig erfolgreicher 
und deutlich kostengünstiger als Interventionsmaßnahmen, wenn schon der Ernstfall eingetreten ist. Es gibt Programme zur Prophylaxe, die an der Förderung von Schutzfaktoren oder an der Verminderung von Risikofaktoren ansetzen.

Folgende Präventionsmaßnahmen zielen auf die Stärkung von Schutzfaktoren ab (Aronson 2001):

- Stärkung des Selbstbewusstseins

- Vermittlung von Selbstwirksamkeitserleben und Erfolgserlebnissen

- Abbau von Schulangst

Als Präventionsmaßnahmen zur Verminderung von Risikofaktoren gelten:

- Zugangskontrolle zu Waffen

- Verbot und Kontrolle von Gewaltdarstellungen

- Verhinderung oder Verminderung von medialen Darstellungen von Amoktaten, um Nachahmungstaten zu verhindern.

Bannenberg (2010b) ist in Bezug auf die Wirksamkeit dieser Maßnahmen eher skeptisch. Ihrer Meinung nach waren die spezifischen Amok-Präventionsmaßnahmen bisher nicht erfolgreich. Erfolg versprechender seien allgemeine Maßnahmen wie die Suizid- oder allgemeine Gewaltprävention gewesen. Zur Klärung dieser Fragen könnte die Arbeitsgruppe um Scheithauer (2008) mit zukünftigen Forschungsergebnissen über Leaking beitragen.

In den USA haben sich jedoch die angewandten Präventionsmaßnahmen schon bewährt. Dies wurde durch den Rückgang von School Shootings bestätigt. Im Vergleich zu den goer-Jahren starben 2008-2009 deutlich weniger Menschen an US-Schulen (Donner 2011).

Ein ausschließlich „kompetitives“ Schulsystem stellt Leistung und damit den Lehrplan in den Mittelpunkt und nicht das Kind als Individuum. Die zentrale Botschaft lautet:

\section{„Nicht du selbst bist wichtig, sondern das Ziel, das du erreichen sollst.“}

Dies führt zu einer immer größer werdenden „sozialen Kälte“ und zu einer zunehmenden Dominanz der „Ellbogengesellschaft“, die letztlich zu Isolation und Einsamkeit führt und sensible Kinder und Jugendliche überfordert und zu ihrer Marginalisierung beitragen kann. Andererseits kann Schule kein „Reparaturbetrieb gesellschaftlicher Delinquenzen“ sein. Sie sind aber eine wichtige Instanz für die Entwicklung sozialer Kompetenzen und für die Umsetzung gewaltpräventiver Maßnahmen.

Eine wichtige Rolle des pädagogischen Auftrags ist dabei die Förderung der Empathie, die Entwicklung eines „Wir-Gefühls“, einer Gruppen-Identität und 
einer adäquaten Lernatmosphäre. Ein „Urvertrauen“ gründet in der Erfahrung, erwünscht zu sein und sich auf die Personen „ohne Wenn und Aber“ verlassen zu können, auf die man existenziell angewiesen ist. Bindungsforscher sprechen hierbei von ,sicherer Bindung“.

Nach Keupp (2010) braucht Lebenskompetenz einen „Vorrat an Lebenskohärenz“. Dies trägt auch zu einer angemessenen Konfliktfähigkeit bei. In diesen Prozess sollten die Eltern verstärkt einbezogen werden, die selbst in wichtigen sozialen Bereichen oft keine eigene Kompetenzen und soziale Ressourcen entwickelt haben. Erfolg versprechend scheint bislang einzig die „sensible Beobachtung des Sozial- und Gewaltverhaltens" gefährdeter oder auffälliger Schüler zu sein. Für den „Raum Schule “ bedeutet dies eine höhere Kapazität an geschultem Personal, das in der Lage ist, Strategien zur Identifizierung und Lösung der Konflikte in der Schule zu entwickeln und umzusetzen.

Theaterprojekte. Zu einem besseren Verständnis der Gewaltdynamik können auch Theaterprojekte beitragen, die sich mit dem Schul-Amok-Problem beschäftigen (Dössel 2010). In der Zwischenzeit gibt es verschiedene Theaterprojekte zur Schul-Amok-Thematik wie das von Juli Zeh "Good Morning Boys and Girls“, geschrieben als Auftragswerk für das Schauspielhaus Düsseldorf, oder das Stück „KomA“ des ,Jungen Schauspiels Hannover" nach einer Textvorlage von Volker Schmidt und Georg Staudacher und „Verloren“ des ,Jungen Schauspielhaus Bochum "nach einer Collage von Emel Aydogdu. Alle Stücke versuchen, mögliche Anzeichen und Ursachen des Schul-Amoklaufs erkennbar oder zumindest denkbar zu machen.

Lehrerfortbildung. Lehrerinnen und Lehrer sollten durch Wahrnehmungsschulungen und Deeskalationstraining befähigt werden, Auffälligkeiten bei Schülern frühzeitig zu erkennen und sich gemeinsam mit dem schulpsychologischen Dienst mit den auffälligen Schülern auseinanderzusetzen. Eventuell notwendige Hausbesuche und Einbeziehung der Eltern erfordert Zeit. Statt populistisch nach Verschärfung von Gesetzen und Verboten zu rufen, sollten neue Wege der „Inklusion, neue Formen der Ansprache und Anschlussfähigkeit“ (Nassehi 2006) versucht und politisch unterstützt werden. 